\title{
High-Temperature Luminescence Quenching of Colloidal Quantum Dots
}

\author{
Yiming Zhao, ${ }^{\dagger}$ Charl Riemersma, ${ }^{\dagger}$ Francesca Pietra, ${ }^{\dagger}$ Rolf Koole, ${ }^{\ddagger}$ Celso de Mello Donegá, ${ }^{\dagger}$ and \\ Andries Meijerink $^{\dagger, *}$ \\ ${ }^{\dagger}$ Condensed Matter and Interfaces, Debye Institute for Nanomaterials Science, Utrecht University, Princetonplein 5, 3584 CC Utrecht, The Netherlands and ${ }^{\ddagger}$ Philips \\ Research Laboratories, High Tech Campus 4, 5656 AE Eindhoven, The Netherlands
}

\begin{abstract}
A high luminescence efficiency is an important property of colloidal quantum dots (QDs), and quantum yields higher than $90 \%$ have been reported for core-shell QDs. ${ }^{1}$ High efficiencies are especially important for application of QDs as luminescent biolabels, ${ }^{2}$ in QD lasers, ${ }^{3}$ in spectral converters for warm white LEDs, ${ }^{4,5}$ electroluminescent devices, ${ }^{6}$ and solar concentrators. ${ }^{7}$ Luminescence efficiencies are strongly temperature-dependent. ${ }^{8}$ Extensive temperature-dependent luminescence studies for colloidal QDs have been conducted at cryogenic temperatures $(0.3-300 \mathrm{~K}) .^{9-15}$ In this temperature region, interesting effects were observed, including a prolonged lifetime below $20 \mathrm{~K}$ related to bright-dark state splitting, ${ }^{11,16}$ thermally activated quenching due to surface defect states, ${ }^{9,10,17}$ and temperature antiquenching assigned to a phase transition in the capping layer. ${ }^{14,15}$ However, the luminescence properties of QDs above room temperature (RT) are hardly investigated, and yet, for most applications in lumi-

ABSTRACT
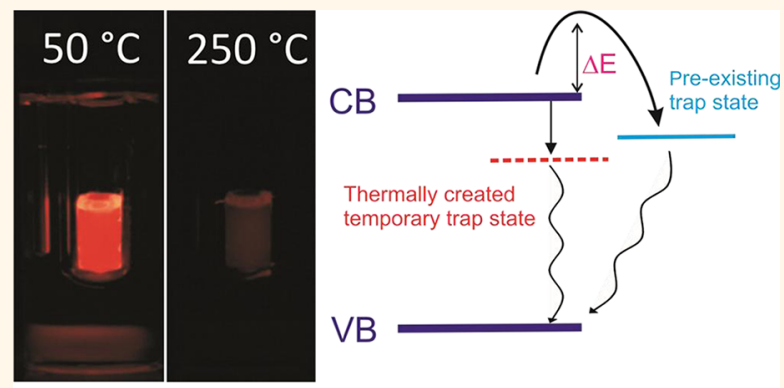

QD in matrix

Thermal quenching of quantum dot (QD) luminescence is important for application in luminescent devices. Systematic studies of the quenching behavior above $300 \mathrm{~K}$ are, however, lacking. Here, high-temperature $(300-500 \mathrm{~K})$ luminescence studies are reported for highly efficient CdSe core-shell quantum dots (QDs), aimed at obtaining insight into temperature quenching of QD emission. Through thermal cycling (yoyo) experiments for QDs in polymer matrices, reversible and irreversible luminescence quenching processes can be distinguished. For a variety of core-shell systems, reversible quenching is observed in a similar temperature range, between 100 and $180^{\circ} \mathrm{C}$. The irreversible quenching behavior varies between different systems. Mechanisms for thermal quenching are discussed.
\end{abstract} nescent devices, the working temperature is higher than $300 \mathrm{~K}$. An interesting example is the recent application of QDs as color converters in warm-white LEDs, ${ }^{18}$ in which QDs serve as narrow band red emitters under excitation with blue light from a (In,Ga)N LED. The narrow emission bandwidth renders QDs superior to classical phosphors based on broad band emission from luminescent ions. ${ }^{19}$ In high-power LEDs for general lighting applications, the heat generated in the $p-n$ junction and phosphor converter layer leads to temperatures as high as $150-200^{\circ} \mathrm{C}$ in the layer applied on top of the blue diode. ${ }^{20}$ To avoid these high temperatures, the QD phosphor layer can be placed in a more remote configuration. Still, temperatures in such a configuration are expected to be well above $50{ }^{\circ} \mathrm{C}$ due to heat dissipation of the QDs themselves (excess energy from converting the blue into red light). Clearly, the quenching
KEYWORDS: quantum dots - luminescence temperature quenching - LED · core-shell nanostructure $\cdot$ quantum yield

of QD luminescence at elevated temperatures is relevant for application of QDs in luminescent devices, and a better insight in the quenching behavior is needed.

Despite its importance, research on luminescence temperature quenching above RT is very limited for QDs. It is theoretically expected for a QD to have a very high luminescence quenching temperature $\left(T_{\mathrm{q}}\right)$. Three generally accepted mechanisms for thermal quenching involve thermally activated crossover from the excited state to the ground state, multiphonon relaxation, and thermally activated photoionization. The first mechanism is generally depicted in a simple configurational coordinate diagram. ${ }^{8,21}$ The energy difference between the minimum *Address correspondence to
a.meijerink@uu.nl.

Received for review July 18, 2012 and accepted September 14, 2012.

Published online September 14, 2012 $10.1021 / \mathrm{nn} 303217 q$

() 2012 American Chemical Society 
of the excited state parabola and the crossing point between the excited state and ground state parabola is considered as activation energy for thermal quenching. For electronic transitions with a small Huang-Rhys factor $S$ (i.e., small electron-phonon coupling), this activation energy is high. For QD luminescence, $S$ has been shown to be very small $(\sim 0.1-0.2)$ from the low relative intensity of the phonon side bands in single dot emission spectra. ${ }^{22}$ For these small values of $S$, temperature quenching is not expected. ${ }^{21}$ Multiphonon relaxation is a quenching process in which the energy gap between the excited state and the ground state is bridged by the simultaneous emission of phonons (vibrational quanta). If the gap can be bridged by 5 phonons, radiative and nonradiative decay compete. For larger gaps, radiative decay dominates and high quantum yields are possible. For QD emission in the red spectral region, at least 50 vibrational quanta are required to bridge the gap due to the low $\mathrm{Cd}-\mathrm{Se}$ vibrational energy, and thus multiphonon relaxation is not expected to play a role. Finally, thermally activated photoionization can cause luminescence quenching by escape of a charge carrier from a luminescent center by thermally activated ionization. In traditional phosphors, the process involves ionization from a localized dopant excited state to the host conduction band. For a $Q D$, it can be viewed as thermally activated escape of a charge carrier to a nearby trap state. Contrary to the other quenching mechanisms, which are intrinsic to the nature of the luminescent center, quenching processes involving traps or defects are not intrinsic, and therefore, the quenching temperature can vary for the same type of luminescent center depending on the nature and location of the defects or traps. Traps and defects have been used to explain luminescence quenching above $50 \mathrm{~K}$ for organically capped $\mathrm{CdSe}^{11}$ and $\mathrm{CdTe}^{10}$ QDs. For core-shell QDs, the quenching temperature increased to $200 \mathrm{~K}$ or above RT for CdSe/ CdS/ZnS multishells ${ }^{12}$ or $\mathrm{CdSe} / \mathrm{CdS}$ nanorods. ${ }^{23}$ These observations suggest that thermal quenching of QDs is related to carrier trapping in (surface) defect states, and surface passivation through inorganic shells can suppress this process. The luminescence quenching behavior of core-shell QDs at elevated temperature has not yet been systematically studied, and therefore, the intrinsic quenching temperature of QDs remains to be determined. In this work, we focus on the high-temperature region (300-500 K) and systematically study the thermal behavior of different types of highly efficient core-shell QDs, aiming at obtaining insight into the origin and mechanism of temperature quenching of colloidal QDs. To reduce the influence from surrounding media, the nanocrystal (NC) samples were immobilized and isolated in a cross-linked polymer matrix. We were able to distinguish the reversible and irreversible quenching effects by recording both photoluminescence $(\mathrm{PL})$ spectra and $\mathrm{PL}$ decay curves

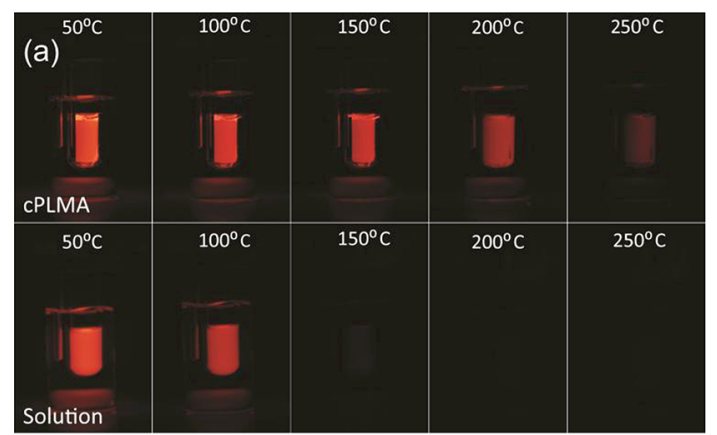

(b)

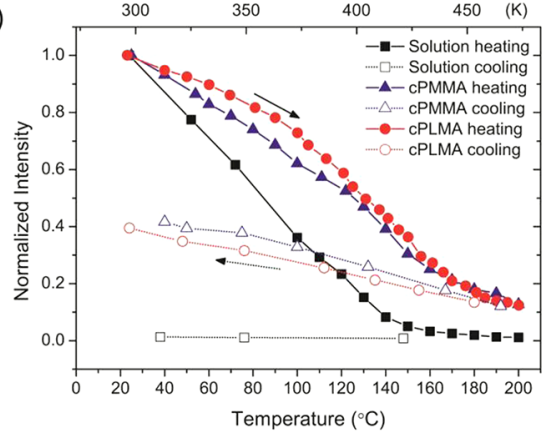

Figure 1. Temperature-dependent photoluminescence (PL) of $\mathrm{CdSe} / \mathrm{CdS} / \mathrm{ZnS}$ core-shell-shell QDs measured in various environments. (a) Photographs taken under UV irradiation at the temperatures indicated. The QDs are incorporated in cross-linked PLMA (top row) or dispersed in ODE solution (bottom row) and sealed in glass tubes under $\mathrm{N}_{2}$. (b) Temperature-dependent PL intensity of $\mathrm{CdSe} / \mathrm{CdS} / \mathrm{ZnS}$ QDs in ODE solution (black squares), cross-linked PMMA (blue triangles), and cross-linked PLMA (red circles). The solid symbols mark the heating stages and the open symbols the cooling stages.

during successive heating and cooling cycles to increasingly higher temperatures (hereafter referred to as "yoyo" measurements) .

\section{RESULTS AND DISCUSSION}

To gain understanding on the quenching behavior of QDs at elevated temperature, it is important to also monitor the role of the environment. To this effect, temperature-dependent PL intensity and PL decay time measurements were performed on QDs in different matrices (liquid and solid). To investigate the influence of the medium, $\mathrm{CdSe} / \mathrm{CdS} / \mathrm{ZnS}$ core-shellshell QD samples were dispersed in the high boiling point solvent octadecene (ODE) or incorporated in solid polymer matrices: cross-linked poly (methyl methacrylate) (CPMMA) or cross-linked poly(lauryl methacrylate) (CPLMA). Both polymers show high transparency and limited deformation in the temperature range investigated (up to $220{ }^{\circ} \mathrm{C}$ ). The temperature dependence of the emission intensity of the QDs was measured in the different matrices and is depicted in Figure 1. With increasing temperature, the PL intensity of the QDs in ODE solution drops significantly faster than that in the solid matrix, and it is almost gone above $150^{\circ} \mathrm{C}(423 \mathrm{~K})$, as clearly shown by the photographs taken during the 
experiment (Figure 1a). Along with the quenching of the excitonic QD emission, a red-shifted emission band appears which is assigned to defect emission. After cooling back to RT, the intensity of the QD emission in ODE does not recover, indicating irreversible quenching. The lifetime measurements for the QD emission confirm the much lower quenching temperature (quenching above $70{ }^{\circ} \mathrm{C}$ ) in solution than in solid matrices (quenching above $110^{\circ} \mathrm{C}$ ) (see Supporting Information, Figure S1). The temperature-dependent emission from QD samples in CPLMA and CPMMA shows a reproducible and similar behavior with higher $T_{\mathrm{q}}$. Both the luminescence intensity and lifetime are partially recovered during the cooling process (detailed behavior in the solid matrices will be discussed later). These results show that $T_{\mathrm{q}}$ is strongly influenced by the surrounding medium. In an ODE solution at elevated temperatures, the irreversible quenching is probably caused by surface states created by loss of capping ligands due to detachment. It is well-known that loss of capping ligands leads to surface states in the forbidden gap, which quench the luminescence of quantum dots. ${ }^{24}$ These dynamic effects in solution occur at relatively low temperatures and obscure the observation of intrinsic quenching processes of QDs and make it necessary to incorporate the QDs in a solid matrix. In a solid matrix, the passivating ligands cannot detach and leave the surface, thus preventing the irreversible quenching mechanism that dominates for QDs in solution at relatively low temperatures. For QDs dispersed in a solid environment (CPMMA or CPLMA), very similar and reproducible quenching behavior is observed. The higher $T_{\mathrm{q}}$ and partially reversible quenching behavior confirm that a solid matrix which immobilizes QDs is a good environment for studying temperature quenching of QD luminescence. The two polymer materials have a very different glass transition temperature $\left(T_{\mathrm{g}}\right)$ and stiffness (without cross-linking, PMMA has a $T_{\mathrm{g}}>100{ }^{\circ} \mathrm{C}$ while PLMA has a $T_{\mathrm{g}}<-65^{\circ} \mathrm{C}$ ). ${ }^{25}$ The similarity of the luminescence quenching indicates that the physical properties of the polymer matrices are not very important.

To better understand the thermal quenching processes, it is important to distinguish between reversible and irreversible quenching. One way to achieve this is to study the reversibility of both the decrease of the luminescence intensity and radiative lifetime upon heating and the recovery upon subsequent cooling. Irreversible quenching is related to a change in the QD structure and is not intrinsic quenching, while the reversible quenching can originate from the QD structure itself and be related to intrinsic quenching processes. To study the quenching processes, we performed "yoyo" measurements on various types of QDs, including organically capped CdSe QDs, CdSe/CdS/ZnS core-shellshell QDs, CdSe/CdS dot core/rod shell nanorods, and $\mathrm{CdTe} / \mathrm{CdSe}$ core/shell QDs. The CdSe core sizes of the

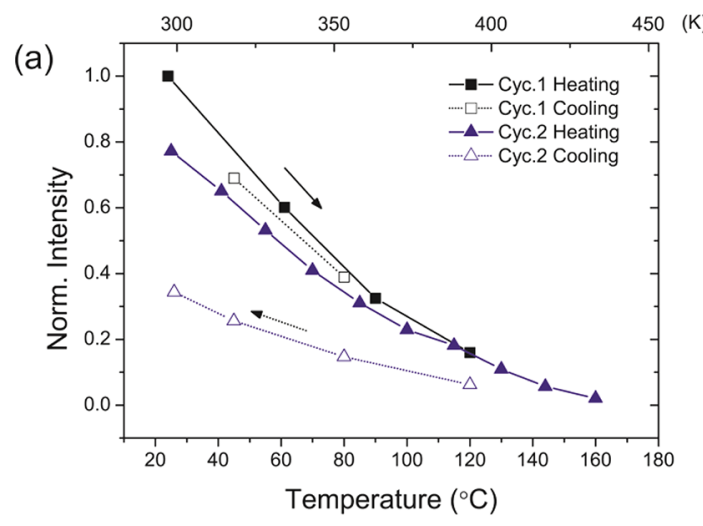

Figure 2. Temperature-dependent (a) intensity and (b) average lifetime of organically capped CdSe QDs incorporated in CPMMA. Data from the first thermal cycle $\left(25-120^{\circ} \mathrm{C}\right)$ are represented by squares, while triangles indicate the second cycle $\left(25-160^{\circ} \mathrm{C}\right)$. Solid symbols refer to the heating process, while cooling process is indicated by open symbols.

structures are similar ( 3.0 to $3.4 \mathrm{~nm})$, and the luminescence quantum yields are high (60-70\%, except for the bare QDs which have a lower QY). The characteristics of the samples are given in the Supporting Information (Table S1). By monitoring the luminescence intensity and lifetime for successive heating and cooling cycles, we are able to differentiate between irreversible and reversible quenching processes and determine the temperature range for quenching.

The initial yoyo measurements are done on CdSe QDs embedded in CPMMA. In Figure 2, the PL intensity and lifetime are plotted as a function of temperature for subsequent heating and cooling cycles. Upon raising the temperature, both the intensity and average lifetime decrease but the decrease in intensity is more pronounced. This result is consistent with previous observations at lower temperatures ${ }^{9,11,12}$ and can be regarded as the higher temperature extension of the quenching process which starts below RT. The cooling measurements reveal that the decrease in lifetime is completely reversible while the PL intensity recovers only partially. There is a $20 \%$ permanent loss of intensity after heating up to $120^{\circ} \mathrm{C}$, and this loss increases to $60 \%$ after heating to $160{ }^{\circ} \mathrm{C}$ in the second cycle. It indicates that part of the dots turns permanently 
(a)
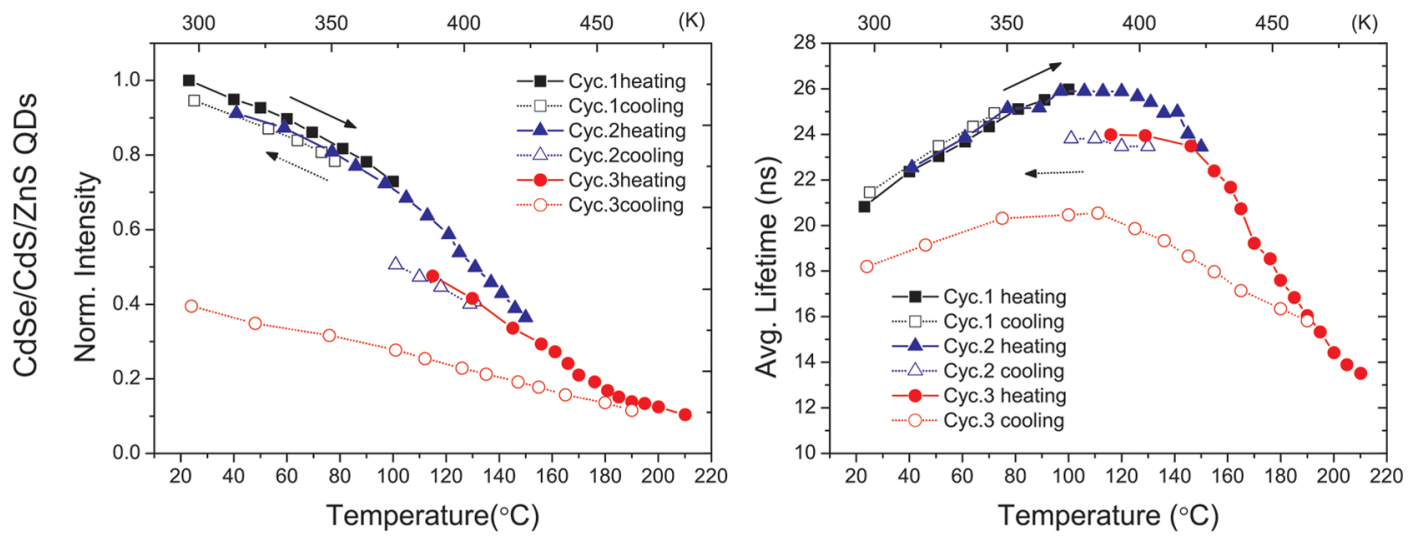

(b)
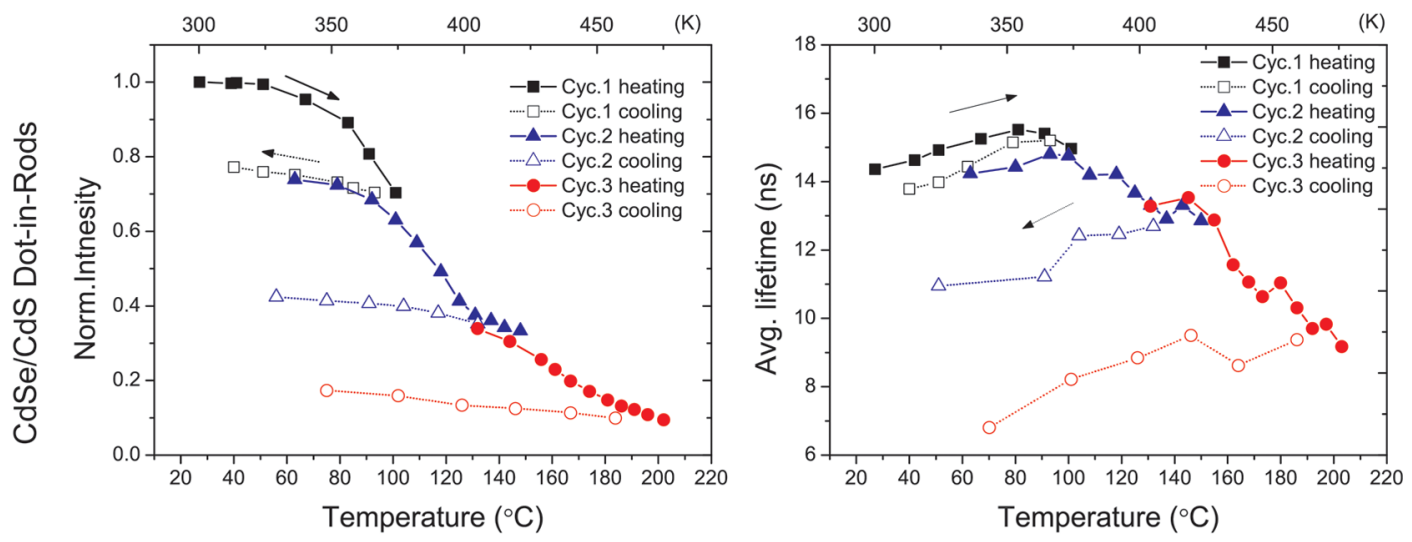

(c)
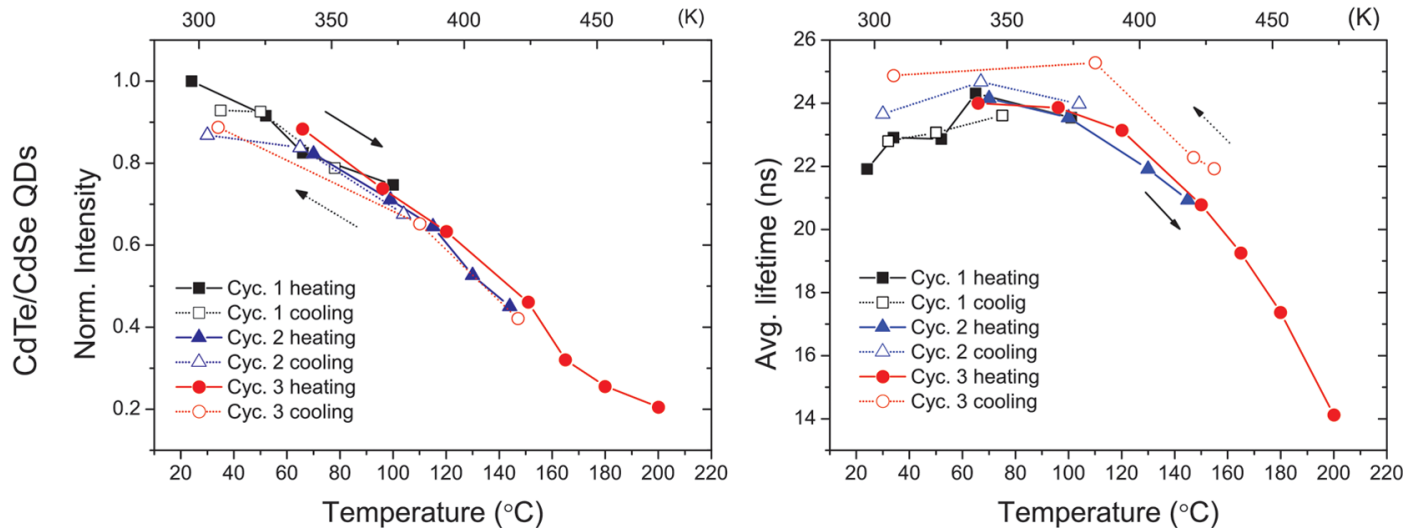

Figure 3. Temperature-dependent PL intensity (left column) and average luminescence lifetime (right column) of (a) CdSe/ $\mathrm{CdS} / \mathrm{ZnS}$ QDs incorporated in CPLMA, (b) CdSe/CdS nanorods incorporated in cPLMA, and (c) CdTe/CdSe incorporated in CPMMA. Squares mark the first thermal cycle $\left(20-100^{\circ} \mathrm{C}\right)$, triangles the second cycle $\left(20-150^{\circ} \mathrm{C}\right)$, and the circles the third cycle $\left(20-210^{\circ} \mathrm{C}\right)$. The solid symbols refer to heating and open symbols to cooling.

"dark" during the heating process. The PL lifetime is biased toward the remaining "bright" QDs, and the complete recovery shows that these are reversibly quenched at high temperatures.

For practical applications, the thermal quenching of $\mathrm{CdSe} / \mathrm{CdS} / \mathrm{ZnS}$ core-multishell QDs is important since these types of dots can be synthesized with high quantum efficiency and stability and is the most commonly used QD structure in applications (biolabels, ${ }^{2}$
QD-LEDs, ${ }^{6}$ and color converters ${ }^{4,26}$ ). We did yoyo experiments in three temperature regions: $25-100^{\circ} \mathrm{C}(\mathrm{I})$, $100-150^{\circ} \mathrm{C}$ (II), and $150-210^{\circ} \mathrm{C}$ (III). The results for the $\mathrm{PL}$ intensity and lifetime measurements are presented in Figure 3a. In region I, up to $100{ }^{\circ} \mathrm{C}$, the total PL intensity decreases while the average lifetime increases slightly. Both changes are fully reversible. These observations show that the PL intensity is not always a good indicator to study the thermal quenching of QDs. 
The overall intensity may decrease due to complete quenching of a fraction of the QDs. The exciton lifetime probes the remaining bright fraction of QDs and is thus a better indicator for intrinsic quenching mechanisms in QDs. The slight increase in lifetime can be explained by thermally activated delocalization of the electron wave function from the CdSe core to the CdS shell due to the shallow offset between the conduction bands of $\mathrm{CdSe}$ and CdS. Further delocalization of the electron wave function reduces the wave function overlap with the hole and has been suggested as an explanation for the increase in lifetime. ${ }^{23}$ An alternative explanation is thermal population of higher energy dark exciton states (for which emission to the ground state is forbidden). The exciton energy level structure of $\mathrm{CdSe}$ QDs has been intensively studied. The shortening in lifetime between 4 and $50 \mathrm{~K}$ can be explained by thermal population of the $\left( \pm 1^{L}\right)$ bright exciton finestructure state situated just above the lowest energy dark exciton state $( \pm 2) .{ }^{11,14}$ Another optically inactive excited state $\left(0^{\mathrm{L}}\right)$ is situated above the bright state $\left( \pm 1^{\mathrm{L}}\right)$ (20 meV higher in energy for $3.1 \mathrm{~nm}$ CdSe QDs). ${ }^{27}$ With increasing temperature, this dark state is thermally populated, and this can also explain the observed lengthening of the lifetime between 20 and $100{ }^{\circ} \mathrm{C}$. The observed red shift of the emission can also contribute to the increase of the lifetime, but based on size-dependent radiative decay rates, only a $\sim 1.4 \mathrm{~ns}$ increase is expected for the observed $10 \mathrm{~nm}$ shift around $600 \mathrm{~nm} .^{15}$ The absence of lifetime shortening gives evidence that there is no intrinsic quenching up to $100{ }^{\circ} \mathrm{C}$.

A strong decrease in both the PL intensity and the exciton lifetime occurs between 100 and $150{ }^{\circ} \mathrm{C}$ (region II). When the $\mathrm{CdSe} / \mathrm{CdS} / \mathrm{ZnS}$ QDs were heated above $100{ }^{\circ} \mathrm{C}$, a steeper decrease in intensity was observed. This change is accompanied by a decrease of lifetime which competes with the lifetime increase process observed in region I. To illustrate the initial lengthening followed by a shortening of the exciton lifetime, several PL decay curves are presented in Figure 4 for different temperatures. The decrease of lifetime starting above $100{ }^{\circ} \mathrm{C}$ is a direct evidence for $\mathrm{TQ}$. The changes of the intensity and lifetime are not totally reversible after heating to $150^{\circ} \mathrm{C}$. Upon cooling to $100^{\circ} \mathrm{C}$, there is also permanent loss of intensity and shortening of lifetime, giving evidence for an irreversible structural change in part of the QD ensemble.

In the last cycle, with heating over $150^{\circ} \mathrm{C}$, the decay of the intensity and lifetime follows the same trend as the decrease in region II until $210{ }^{\circ} \mathrm{C}$, where the intensity is less than $10 \%$ of the initial value. During cooling, there is a partial recovery for both intensity and lifetime. In this region, the TQ quenching process clearly contains an irreversible and a reversible part. There is also a continuous red shift of the PL peak with increasing temperature, which is consistent with

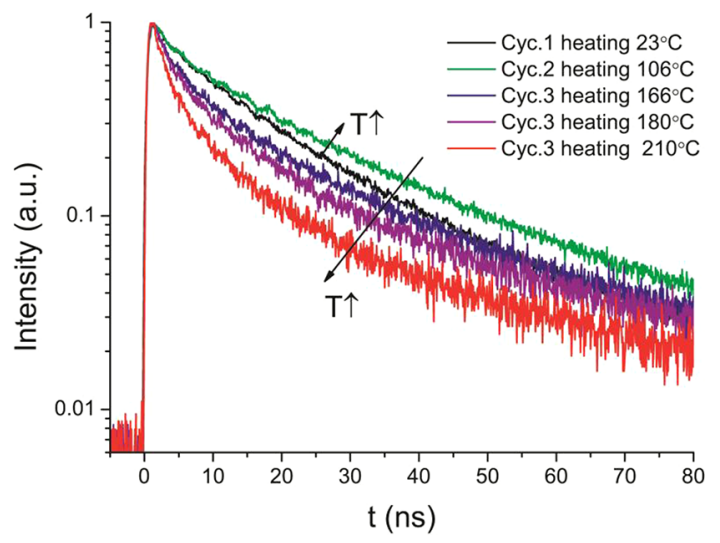

Figure 4. PL decay curves of $\mathrm{CdSe} / \mathrm{CdS} / \mathrm{ZnS}$ core-shellshell QDs in CPMMA for excitation at $406 \mathrm{~nm}$ and emission at $\sim 600 \mathrm{~nm}$ at the temperatures indicated.

low-temperature studies where the shift in band gap is described by the Varshni equation., ${ }^{9,12}$ The shift is totally reversible (Supporting Information, Figures S2 and S3).

A third system that was investigated in the present work is the $\mathrm{CdSe} / \mathrm{CdS}$ dot core/rod shell nanorods. The unique optical properties of these nanostructures include a high QY, polarized absorption and emission, and suppression of blinking and Auger recombination. On the basis of these characteristics, they have been proposed as materials for opto-electronic devices. ${ }^{23}$ We performed yoyo cycling experiments in three temperature regions for $\mathrm{CdSe} / \mathrm{CdS}$ dot core/rod shell nanorods (core diameter $=3.2 \mathrm{~nm}$, rod length $=$ $\sim 20 \mathrm{~nm}$ ) embedded in cPLMA. The temperaturedependent $P L$ intensity and average lifetime are plotted in Figure $3 \mathrm{~b}$. Up to $80^{\circ} \mathrm{C}$, the intensity is constant while the lifetime shows a small increase upon heating. This result is consistent with measurements at lower temperatures, ${ }^{23}$ where between 70 and $300 \mathrm{~K}$ an increase of lifetime and a constant intensity were reported. Upon raising the temperature above $80^{\circ} \mathrm{C}$, the intensity decreases and the lifetime becomes shorter (Supporting Information, Figure S4). These changes are irreversible as both the intensity and lifetime do not recover after cooling. In the second and third cycle, where the nanorods were heated to 150 and $200{ }^{\circ} \mathrm{C}$, a similar trend is observed: an irreversible decrease of intensity and lifetime occur with increasing temperature. At $200{ }^{\circ} \mathrm{C}$, the intensity has dropped below $10 \%$ of the initial value and hardly recovers upon subsequent cooling (Figure $3 \mathrm{~b}$ ). During heating to high temperatures, a broad defect-related emission band also appears at longer wavelength (Supporting Information, Figure S5).

The final nanostructures investigated are $\mathrm{CdTe} / \mathrm{CdSe}$ core-shell QDs. Depending on the thickness of the core and shell, a type-II band alignment can be realized for the $\mathrm{CdTe} / \mathrm{CdSe}$ system, ${ }^{28}$ but for the presently studied system ( $3 \mathrm{~nm}$ core, $1.3 \mathrm{~nm}$ shell), the short luminescence lifetime $(\sim 26 \mathrm{~ns}$ at RT) indicates that 
there is strong delocalization of the electron over the hetero-nanostructure (i.e., the QDs are still in the type$1^{1 / 2}$ or "quasi" type-Il localization regime ${ }^{28,29}$ ). The results of the yoyo measurements on $\mathrm{CdTe} / \mathrm{CdSe} \mathrm{QDs}$ embedded in CPMMA are presented in Figure $3 \mathrm{c}$ for three temperature ranges (RT-100 ${ }^{\circ} \mathrm{C}, 100-150{ }^{\circ} \mathrm{C}$, and $150-200^{\circ} \mathrm{C}$ ). Similar to the observations for other systems, in all three temperature regions, the intensity decreases with the strongest decrease between 100 and $160{ }^{\circ} \mathrm{C}$. The behavior of the lifetime is also analogous to other systems: a slight increase between RT and $80^{\circ} \mathrm{C}$ is followed by a decrease above $100{ }^{\circ} \mathrm{C}$. However, different from the systems described above, both the PL intensity and the exciton lifetime fully recover upon cooling, indicating that the temperature quenching in the $\mathrm{CdTe} / \mathrm{CdSe}$ core/shell QDs is totally reversible up to $200^{\circ} \mathrm{C}$. This observation is important. It reveals that irreversible quenching is not necessarily present in every QD structure and that, depending on the band structure and/or core-shell heterointerface, the irreversible formation of surface/interface or defect states which quench the luminescence can be avoided.

The thermal quenching studies for the luminescence of four different QD systems show several general trends. A decrease in intensity is observed starting above RT. Initially the decrease is slow, and a more rapid drop in intensity occurs for all systems studied between 100 and $150^{\circ} \mathrm{C}$. For the core-shell structures, the luminescence lifetime shows a slight increase between RT and $\sim 80^{\circ} \mathrm{C}$, while for organically capped CdSe QDs, no increase in luminescence lifetime is observed. Between 100 and $180^{\circ} \mathrm{C}$, a clear shortening of the lifetime is observed for all QD structures, along with a strong decrease in emission intensity. The reversibility of the drop in emission intensity and reduction of the lifetime in heating/cooling cycles varies: for $\mathrm{CdTe} / \mathrm{CdSe} \mathrm{QDs}$, the intensity and lifetime fully recover upon cooling, while for CdSe cores in CdS nanorods, the loss in intensity is mostly permanent. For the CdSe QDs and CdSe/CdS/ZnS core-shell-shell QD structures, both reversible and irreversible quenching contribute. In earlier work on luminescence quenching below RT, luminescence quenching is usually ascribed to thermally activated carrier trapping. ${ }^{11}$ Surface states, defects, or impurities in the immediate surroundings of the QD can act as trapping centers, and carrier trapping is followed by nonradiative recombination or red-shifted defect-related emission. The present observations for highly efficient core-shell structures with RT quantum yields of $60-70 \%$ show an onset for temperature quenching around $100{ }^{\circ} \mathrm{C}$. This temperature is significantly higher than that for organically capped CdSe QDs, which are already partially quenched at RT. This clearly demonstrates the importance of inorganic shell coating for confining the wave functions, eliminating the surface defect states and suppressing the surface trapping states, hence improving the QD's hightemperature optical performance. (a)

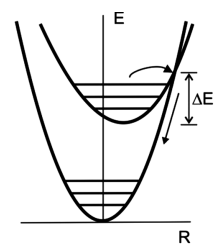

(c)

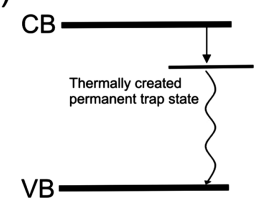

(b)

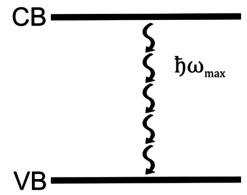

(d)

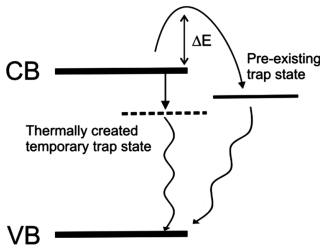

Figure 5. Schematic representation of luminescence quenching mechanisms. Two traditional models for intrinsic quenching of luminescence are based on (a) thermally activated crossover and (b) multiphonon relaxation. Quenching related to traps are shown for (c) irreversible quenching, which is related to thermally created permanent trap states and (d) reversible quenching, which is related to thermally created temporary trap states or thermally activated trapping processes involving pre-existing trap states.

The yoyo measurements reveal details and differences for the thermal behavior of the core-shell structures, giving insight into the origin of thermal quenching. Two different thermal quenching processes can be distinguished: irreversible and reversible quenching. In Figure 5, different quenching mechanisms are schematically depicted. Since the irreversible quenching behavior varies between different structures and complicates the interpretation, we consider it first. For CdSe/CdS/ZnS QDs and CdSe cores embedded in CdS nanorods, the average PL lifetime and $\mathrm{PL}$ intensity start to decrease sharply, after heating above $\sim 100{ }^{\circ} \mathrm{C}$. The PL intensity drop and lifetime shortening are only partially reversible. After heating to $210^{\circ} \mathrm{C}$ and cooling to $\mathrm{RT}$, there is a $60 \%$ permanent loss of intensity for CdSe/CdS/ZnS QDs and over $80 \%$ for $\mathrm{CdSe}$ cores embedded in CdS nanorods. The most likely origin of this irreversible quenching is the formation of surface states or defects related to an irreversible structural change (Figure $5 \mathrm{c}$ ). The thermally induced irreversible structural changes are possibly due to the lattice mismatch between core and shell materials, which generate interfacial strain and favor dislocation of atoms. Upon raising the temperature, the difference in expansion of core and shell materials and increased mobility of the atoms may result in the formation of temporary or permanent trapping centers. One may argue that structural changes at these temperatures is unexpected since the synthesis temperatures for the QDs are much higher, typically between 200 and $350{ }^{\circ} \mathrm{C}$, and yet high QYs are realized. However, the synthesis of core-shell QDs (or nanorods) is carried out under a high chemical potential environment with a large excess of surrounding ligands and precursors, resulting in high-quality QDs. ${ }^{29}$ It is known that after long time annealing at elevated temperature 
in the reaction mixture, the QY decreases ${ }^{30}$ and the shape of QDs and nanorods may also change upon heating. ${ }^{29,31}$ Therefore, local structural changes for core-shell dots (or rods) can be expected during thermal cycling. Surface states and defects created in the shell material may also quench the luminescence due to delocalization of charge carriers over the shell. The strongly irreversible quenching observed for the $\mathrm{CdSe} / \mathrm{CdS}$ nanorods may be related to the large surface area of the rods, which increases the probability for the creation of a defect or surface state in the $\mathrm{CdS}$ nanorod which can trap the delocalized charge carrier.

From a practical point of view, the irreversible quenching can be a crucial limiting factor for applications of core-shell nanostructures at elevated temperatures. Interestingly, the results on $\mathrm{CdTe} / \mathrm{CdSe}$ core/ shell QDs indicate that the irreversible quenching process is absent in this system. During the yoyo measurements in the whole temperature region up to $200{ }^{\circ} \mathrm{C}$, the intensity and lifetime variation is totally reversible. Interfacial diffusion between Se and Te may reduce the interfacial strain. Chin et al. ${ }^{32}$ previously reported a slight antiquenching behavior in the range of 300-360 K for CdTe/CdSe core-shell nanostructures with core size and emission wavelength comparable to those investigated here. This indicates that, depending on the sample, the QY may even increase due to thermal annealing effects. The results demonstrate that $\mathrm{CdTe} /$ CdSe hetero-nanocrystals are interesting materials for high-temperature lighting applications.

Reversible luminescence quenching may be related to intrinsic quenching. Traditional luminescent materials applied in lighting show fully reversible temperature quenching due to thermally activated crossover from the excited state to the ground state (Figure 5a) or multiphonon relaxation (Figure $5 b$ ), as discussed above. The quenching is an intrinsic and characteristic property of the luminescent species. For the coreshell QDs, reversible quenching is observed between 100 and $180{ }^{\circ} \mathrm{C}$ and is most reliably observed in the temperature dependence of the luminescence decay time. The temperature range is similar for the different core-shell structures investigated. It is unlikely that the quenching is due to one of the two traditional reversible quenching processes. The electron-phonon coupling is weak for excitonic emission and is reflected by a small Huang-Rhys parameter $S$ (for CdSe, $S$ is about $0.1-0.2) .^{33}$ In the configurational coordinate diagram, the offset in equilibrium distance between the ground state and excited state parabola is very small. Hence the crossing point is at very high energies, and for systems with a Huang-Rhys parameter below 1, no thermal quenching due to thermally activated crossover has been observed.

A quenching mechanism for luminescent species characterized by a small parabola offset is multiphonon relaxation, where the energy gap is bridged by the simultaneous emission of phonons ${ }^{8,34}$ (Figure 5b). The nonradiative multiphonon relaxation rate decreases exponentially with the number of phonons $p$ (with $p=\Delta E / \hbar \omega_{\max }$, where $\Delta E$ is the energy gap and $\hbar \omega_{\max }$ is the maximum phonon energy). Multiphonon relaxation can compete with radiative decay if the energy gap can be bridged by $\sim 5$ phonons. If more than 5 phonons are required, radiative decay dominates, while for less than 5 phonons, nonradiative decay dominates. For the presently studied systems with $\sim 3-3.5 \mathrm{~nm}$ CdSe cores, the band gap $(\Delta E)$ is about $2 \mathrm{eV}$ and the LO phonon energies for CdSe are $\sim 25 \mathrm{meV}$, thus $p=80$, which indicates that the gap is too large for effective multiphonon relaxation. Coupling with highenergy vibrations of surrounding ligands (e.g., $\mathrm{C}-\mathrm{H}$ or $\mathrm{N}-\mathrm{H}$ stretching vibrations of $\sim 0.4 \mathrm{eV}$ ) can give rise to multiphonon relaxation $(p=5)$ but cannot explain the temperature dependence. The multiphonon relaxation rate is only enhanced at higher temperatures if the phonon occupation number or Bose-Einstein factor $n\left(n=1 /\left(e^{\hbar \omega / k T}\right)-1\right)$ increases, which can be viewed as stimulated multiphonon emission in the presence of a "phonon field", similar to stimulated photon emission. ${ }^{21,35}$ For high-energy $0.4 \mathrm{eV}$ vibrations, there is no thermal population ( $n$ close to 0 ) below $1000 \mathrm{~K}$, and multiphonon relaxation can therefore not explain the observed temperature dependence. The large distance to the high-energy ligand vibrations, especially for core-shell systems where the ligands are on the surface of the shell, also reduces the coupling strength (which scales with $R^{-6}$ ) and makes multiphonon relaxation highly unlikely.

As discussed above, the two traditional models for intrinsic quenching of luminescent materials cannot explain the reversible quenching of luminescence in QDs. An alternative explanation is thermally activated creation of surface states or defects which trap the charge carriers (Figure $5 \mathrm{~d}$ ), similar to the process explaining the irreversible quenching. However, upon cooling, the thermally created traps relax back and the original configuration with high quantum yields is restored. The creation of trap states is induced by ion mobility at elevated temperatures. Ion exchange experiments on $\mathrm{CdSe}$ and $\mathrm{PbSe} \mathrm{QDs}$ at temperatures between RT and $100{ }^{\circ} \mathrm{C}$ show that the ions in these semiconductor QDs are mobile at relatively low temperatures, ${ }^{36,37}$ and thus the creation of surface (interface) states and defects by local changes in the QD structure at temperatures between 100 and $200^{\circ} \mathrm{C}$ can be due to displacement of (surface) atoms. The temperature range in which reversible quenching takes place is similar, between 100 and $180{ }^{\circ} \mathrm{C}$, for the different types of 3-3.5 nm CdSe core-shell structures, which is consistent with the formation of defects due to increased ionic mobility in the CdSe QDs at similar elevated temperatures. For the $\mathrm{CdTe} / \mathrm{CdSe}$ QDs, anion disordering at the core/shell interface 
facilitates relaxation upon cooling. An Arrhenius fit describes the observed temperature dependence of the luminescence intensity well and yields a thermal activation energy of $\sim 0.3-0.4 \mathrm{eV}$ (Supporting Information, Figure S6 and Table S2). This activation energy is about 10 times larger than activation energies between 10 and $70 \mathrm{meV}$ reported for luminescence temperature quenching in $\mathrm{CdSe}$ in the low-temperature regime. ${ }^{38-40}$ A similar activation energy $(0.24 \mathrm{eV})$ was recently reported as activation energy for enhanced blinking in excitation wavelength-dependent blinking studies. ${ }^{41}$ For the CdSe/CdS/ZnS core-shell-shell QDs and CdSe cores embedded in CdS nanorods, the thermally induced mobility gives rise to the formation of trap states which do not relax back upon cooling. Finally, part of the reversible quenching may also be related to thermally activated carrier escape to pre-existing trap states. This mechanism is usually invoked to explain low-temperature quenching processes (below $200 \mathrm{~K}$ ) where the creation of trap states is unlikely. Thermally activated escape of charge carriers can explain the observed decrease in emission intensity. Naturally, this process is fully reversible, and the high quantum yield is restored upon cooling. For the presently observed high-temperature quenching, thermally activated carrier escape with high activation energy may also contribute to the observed reversible quenching.

The present observations have revealed a universal quenching behavior for 3-3.5 $\mathrm{nm}$ CdSe QDs above $100{ }^{\circ} \mathrm{C}$ with contributions from reversible and irreversible processes. Further research is required to obtain deeper insight into the exact mechanism for quenching. Different types (e.g., InP and PbSe) and different sizes of core-shell structures need to be investigated to relate the observed trends in the quenching behavior to material properties and particle size. Electron paramagnetic resonance (with and without illumination) may be used to monitor the thermally induced creation of paramagnetic defects. On the basis of insights obtained from these studies, QDs with higher quenching temperatures may be realized, which is especially important in optical applications where the temperature is increased above $100^{\circ} \mathrm{C}$.

\section{CONCLUSIONS}

In conclusion, we have investigated the temperaturedependent photoluminescence intensity and emission lifetime of three representative core-shell CdSe QD systems at high temperatures (RT-200 ${ }^{\circ} \mathrm{C}$ ). Different types of highly efficient (60-70\% QY) CdSe QD systems emitting in the red spectral region and incorporated in a cross-linked polymer matrix were studied. Thermal cycling (yoyo) experiments make it possible to differentiate between reversible and irreversible luminescence quenching processes. Irreversible quenching originates from thermally induced permanent structural changes, giving rise to trap states, and was found in $\mathrm{CdSe} / \mathrm{CdS} / \mathrm{ZnS}$ core-shell-shell QDs and CdSe/CdSe dot core/rod shell nanorods but not in $\mathrm{CdTe} / \mathrm{CdSe}$ core/shell QDs. Reversible quenching was observed to contribute to luminescence quenching for QDs in all three core-shell systems in a similar temperature range (quenching between 100 and $180^{\circ} \mathrm{C}$ ). Reversible quenching is explained by either thermally activated escape of carriers to existing (surface) trap states and/or the thermally activated creation of trap states which relax upon cooling. The quenching temperature for QD emission estimated based on classical models for luminescence quenching (thermally activated crossover or multiphonon relaxation) is much higher than $200^{\circ} \mathrm{C}$ and cannot explain the observed quenching behavior. The results have important implications for application of QDs in optical devices (e.g., warm white LEDs, electroluminescent devices, and QD lasers) with operating temperatures above $100{ }^{\circ} \mathrm{C}$. Further studies on different QD systems are required to gain further insight in the quenching mechanism and to find efficient QDs with higher luminescence quenching temperatures.

\section{METHODS}

Synthesis of Organically Capped Colloidal Quantum Dots (QDs) and Nanorods. Colloidal CdSe QDs capped by trioctylphosphineoxide and hexadecylamine were synthesized following a procedure reported in the literature. ${ }^{30}$ The diameter of the CdSe QDs was estimated from a sizing curve relating the first exciton peak energy with the nanocrystal size. ${ }^{15}$ High-quality $\mathrm{CdSe} /$ $\mathrm{CdS} / \mathrm{ZnS}$ core-shell-shell QDs were prepared according to the one-pot multistage method reported by Lim et al. ${ }^{26}$ The core diameter was $3.4 \mathrm{~nm}$, and after overcoating with about 3 monolayers of $\mathrm{CdS}$ and 2 monolayers of $\mathrm{ZnS}$, the particle size increased to $7 \mathrm{~nm}$. CdSe/CdS dot core/rod shell nanorods were synthesized following the procedure described by Manna et al., ${ }^{42}$ using CdSe QDs with $3.2 \mathrm{~nm}$ diameter seeds. For CdS shell growth, octadecylphosphonic acid (ODPA) was used as the only capping ligand, as it results in better reaction control and favors the formation of nanorods. The nanorods obtained are $21 \mathrm{~nm}$ long and $5.5 \mathrm{~nm}$ in diameter (aspect ratio $=4$ ), as determined by TEM measurements. CdTe/CdSe core-shell
QDs were synthesized as described by Zhang et al. ${ }^{43}$ The CdTe cores were $3.4 \mathrm{~nm}$ in diameter, and the final particle diameter increased to $6.1 \mathrm{~nm}$ after overcoating with the CdSe shell. Photoluminescence (PL) spectra and PL quantum yields were determined on an Edinburgh Instruments FL920 spectrofluorimeter equipped with an integrating sphere.

Incorporation of QDs or Nanorods into Polymer Matrices. In order to immobilize and isolate the nanocrystal (NC) dispersion, CdSe QDs, CdSe/CdS/ZnS core-shell-shell QDs, CdSe/CdS dot core/ rod shell nanorods, and CdTe/CdSe core/shell QDs were incorporated in a cross-linked poly(methyl methacrylate) (CPMMA) or cross-linked poly(lauryl methacrylate) (cPLMA) matrix through a bulk polymerization method. ${ }^{44}$ The procedure starts with washing the NCs twice by precipitation and centrifugation from a chloroform/acetone solution, followed by redispersion in chloroform. The clear NC solution was dried under vacuum to remove the solvent, and the NCs were subsequently redispersed in a monomer mixture. The NC concentration in the matrix was kept low to avoid self-absorption. The monomer 
used for the CPLMA (P(LMA-Co-EGDMA)) matrix was 80 wt \% lauryl methacrylate (LMA) and $20 \mathrm{wt} \%$ ethylene glycol dimethacrylate (EGDMA) as cross-linker; for CPMMA (P(MMA-co-EGDMA)) matrix, 97 wt \% methyl methacrylate (MMA) and 3 wt \% EGDMA were used. For photoinitiated polymerization, 0.25 wt \% photoinitiator, 2-hydroxy-2-methylpropiophenone (97\%, Aldrich), was added to the solution, and $2 \mathrm{~mL}$ of the reaction mixture was loaded into a glass tube and sealed under $\mathrm{N}_{2}$ atmosphere. The glass tube was illuminated by two $360 \mathrm{~nm}$ UV lamps from both sides for $1 \mathrm{~h}$. The polymerization can also be thermally initialized. In that case, 0.3 wt \% thermal initiator, AIBN (Janssen Chimica, recrystallized from ethanol before use), was added into the monomer solution. The sealed tube was then embedded in water bath at $70{ }^{\circ} \mathrm{C}$ for overnight curing.

Temperature-Dependent PL Spectra and Exciton Lifetime Measurements. For temperature-dependent PL measurements, the sample sealed in a glass tube was immersed in a transparent paraffin oil bath in a Pyrex glass container. The bath was heated on a heating plate, and the temperature was controlled by a thermocouple immersed in the paraffin bath at the same height as the excitation laser beam. Photoluminescence spectra were measured under excitation at $406 \mathrm{~nm}$ by a PicoQuant PDL $800-\mathrm{B}$ pulsed diode laser. The emitted light was collected through an optical fiber and dispersed in a $0.3 \mathrm{~m}$ monochromator (Acton Pro SP-300i, 150 lines $/ \mathrm{mm}$, blazed at $500 \mathrm{~nm}$ ) and detected by a liquid nitrogen cooled Princeton Instruments CCD camera (1024 $\times 256$ pixels). PL decay curves were recorded using a PicoQuant time-correlated single-photon counting setup, with the same excitation scheme as described above, operating at a $2.5 \mathrm{MHz}$ repetition rate. The emission was collected using another optical fiber and dispersed by a $0.1 \mathrm{~m}$ monochromator (1350 lines $/ \mathrm{mm}$ blazed at $500 \mathrm{~nm}$ ). A fast Hamamatsu photomultiplier tube (H5738P-01) and a Time Harp 200 time-correlated photon counting card were used for detection and analysis. Care was taken to prevent effects of interpulse pile-up by keeping the photon count rate well below $5 \%$ of the laser repetition rate. At each temperature, first PL spectra were recorded after which a PL decay curve was obtained at the wavelength of emission maximum. The average lifetime values were calculated from the PL decay curve through a Gaussian distribution fitting in PicoQuant FluoFit 3.3.

Conflict of Interest: The authors declare no competing financial interest.

Acknowledgment. This work was financially supported by the division of Chemical Sciences (CW) of the Dutch Science Foundation (NWO) under grant number ECHO.06.B.047.

Supporting Information Available: Additional table and figures. This material is available free of charge via the Internet at http://pubs.acs.org.

\section{REFERENCES AND NOTES}

1. Coe-Sullivan, S. Quantum Dot Developments. Nat. Photonics 2009, 3, 315-316.

2. Skajaa, T.; Zhao, Y.; van den Heuvel, D. J.; Gerritsen, H. C.; Cormode, D. P.; Koole, R.; van Schooneveld, M. M.; Post, J. A.; Fisher, E. A.; Fayad, Z. A.; et al. Quantum Dot and Cy5.5 Labeled Nanoparticles To Investigate Lipoprotein Biointeractions via Förster Resonance Energy Transfer. Nano Lett. 2010, 10, 5131-5138.

3. Klimov, V. I.; Mikhailovsky, A. A.; Xu, S.; Malko, A.; Hollingsworth, J. A.; Leatherdale, C. A.; Eisler, H. J.; Bawendi, M. G. Optical Gain and Stimulated Emission in Nanocrystal Quantum Dots. Science 2000, 290, 314-317.

4. Jang, E.; Jun, S.; Jang, H.; Lim, J.; Kim, B.; Kim, Y. White-LightEmitting Diodes with Quantum Dot Color Converters for Display Backlights. Adv. Mater. 2010, 22, 3076-3080.

5. Lee, J.; Sundar, V. C.; Heine, J. R.; Bawendi, M. G.; Jensen, K. F. Full Color Emission from II-VI Semiconductor Quantum Dot-Polymer Composites. Adv. Mater. 2000, 12, 13111311.

6. Cho, K.-S.; Lee, E. K.; Joo, W.-J.; Jang, E.; Kim, T.-H.; Lee, S. J.; Kwon, S.-J.; Han, J. Y.; Kim, B.-K.; et al. High-Performance
Crosslinked Colloidal Quantum-Dot Light-Emitting Diodes. Nat. Photonics 2009, 3, 341-345.

7. Bomm, J.; Buchtemann, A.; Chatten, A. J.; Bose, R.; Farrell, D. J.; Chan, N. L. A.; Xiao, Y.; Slooff, L. H.; Meyer, T.; Meyer, A.; et al. Fabrication and Full Characterization of State-of-theArt Quantum Dot Luminescent Solar Concentrators. Sol. Energy Mater. Sol. Cells 2011, 95, 2087-2094.

8. Blasse, G.; Grabmaier, B. C. Luminescent Materials; Springer-Verlag: Berlin, 1994.

9. Valerini, D.; Cretí, A.; Lomascolo, M.; Manna, L.; Cingolani, R.; Anni, M. Temperature Dependence of the Photoluminescence Properties of Colloidal CdSe/ZnS Core/Shell Quantum Dots Embedded in a Polystyrene Matrix. Phys. Rev. B 2005, 71, 235409.

10. Morello, G.; De Giorgi, M.; Kudera, S.; Manna, L.; Cingolani, R.; Anni, M. Temperature and Size Dependence of Nonradiative Relaxation and Exciton-Phonon Coupling in Colloidal CdTe Quantum Dots. J. Phys. Chem. C 2007, 111, 5846-5849.

11. Donegá, C. d. M.; Bode, M.; Meijerink, A. Size- and Temperature-Dependence of Exciton Lifetimes in CdSe Quantum Dots. Phys. Rev. B 2006, 74, 085320.

12. Jing, P.; Zheng, J.; Ikezawa, M.; Liu, X.; Lv, S.; Kong, X.; Zhao, J.; Masumoto, Y. Temperature-Dependent Photoluminescence of CdSe-Core CdS/CdZnS/ZnS-Multishell Quantum Dots. J. Phys. Chem. C 2009, 113, 13545-13550.

13. Jones, M.; Lo, S. S.; Scholes, G. D. Quantitative Modeling of the Role of Surface Traps in CdSe/CdS/ZnS Nanocrystal Photoluminescence Decay Dynamics. Proc. Natl. Acad. Sci. U.S.A. 2009, 106, 3011-3016.

14. Crooker, S. A.; Barrick, T.; Hollingsworth, J. A.; Klimov, V. I. Multiple Temperature Regimes of Radiative Decay in CdSe Nanocrystal Quantum Dots: Intrinsic Limits to the DarkExciton Lifetime. Appl. Phys. Lett. 2003, 82, 2793-2795.

15. Donegá, C. d. M.; Koole, R. Size Dependence of the Spontaneous Emission Rate and Absorption Cross Section of CdSe and CdTe Quantum Dots. J. Phys. Chem. C 2009, 113, 6511-6520.

16. Wuister, S. F.; de Mello Donegá, C.; Meijerink, A. Luminescence Temperature Antiquenching of Water-Soluble CdTe Quantum Dots: Role of the Solvent. J. Am. Chem. Soc. 2004, 126, 10397-10402.

17. Wuister, S. F.; van Houselt, A.; Donegá, C. d. M.; Vanmaekelbergh, D.; Meijerink, A. Temperature Antiquenching of the Luminescence from Capped CdSe Quantum Dots. Angew. Chem., Int. Ed. 2004, 43, 3029-3033.

18. Nizamoglu, S.; Erdem, T.; Sun, X. W.; Demir, H. V. WarmWhite Light-Emitting Diodes Integrated with Colloidal Quantum Dots for High Luminous Efficacy and Color Rendering. Opt. Lett. 2010, 35, 3372-3374.

19. Lin, C. C.; Liu, R.-S. Advances in Phosphors for LightEmitting Diodes. J. Phys. Chem. Lett. 2011, 2, 1268-1277.

20. Bachmann, V.; Ronda, C.; Meijerink, A. Temperature Quenching of Yellow $\mathrm{Ce}^{3+}$ Luminescence in YAG:Ce. Chem. Mater. 2009, 21, 2077-2084.

21. Henderson, B.; Imbusch, G. F. Optical Spectroscopy of Inorganic Solids; Oxford University Press: London, 2006.

22. Empedocles, S. A.; Norris, D. J.; Bawendi, M. G. Photoluminescence Spectroscopy of Single CdSe Nanocrystallite Quantum Dots. Phys. Rev. Lett. 1996, 77, 3873-3876.

23. Rainòmgrt, G.; Stöferle, T.; Moreels, I.; Gomes, R.; Kamal, J. S.; Hens, Z.; Mahrt, R. F. Probing the Wave Function Delocalization in CdSe/CdS Dot-in-Rod Nanocrystals by Time- and Temperature-Resolved Spectroscopy. ACS Nano 2011, 5, 4031-4036.

24. Bullen, C.; Mulvaney, P. The Effects of Chemisorption on the Luminescence of CdSe Quantum Dots. Langmuir 2006, 22, 3007-3013.

25. Rogers, S.; Mandelkern, L. Glass Transitions of the Poly(n-alkyl methacrylates). J. Phys. Chem. 1957, 61, 985-991.

26. Lim, J.; Jun, S.; Jang, E.; Baik, H.; Kim, H.; Cho, J. Preparation of Highly Luminescent Nanocrystals and Their Application to Light-Emitting Diodes. Adv. Mater. 2007, 19, 1927-1932.

27. Efros, A. L.; Rosen, M.; Kuno, M.; Nirmal, M.; Norris, D. J.; Bawendi, M. Band-Edge Exciton in Quantum Dots of 
Semiconductors with a Degenerate Valence Band: Dark and Bright Exciton States. Phys. Rev. B 1996, 54, 48434856.

28. Donegá, C. d. M. Formation of Nanoscale Spatially Indirect Excitons: Evolution of the Type-II Optical Character of CdTe/CdSe Heteronanocrystals. Phys. Rev. B 2010, 81, 165303.

29. Donegá, C. d. M. Synthesis and Properties of Colloidal Heteronanocrystals. Chem. Soc. Rev. 2011, 40, 1512-1546.

30. Donegá, C. d. M.; Hickey, S. G.; Wuister, S. F.; Vanmaekelbergh, D.; Meijerink, A. Single-Step Synthesis To Control the Photoluminescence Quantum Yield and Size Dispersion of CdSe Nanocrystals. J. Phys. Chem. B 2003, 107, 489496.

31. Grodzinska, D.; Pietra, F.; van Huis, M. A.; Vanmaekelbergh, D.; Donegá, C. d. M. Thermally Induced Atomic Reconstruction of PbSe/CdSe Core/Shell Quantum Dots into PbSe/ CdSe Bi-hemisphere Hetero-nanocrystals. J. Mater. Chem. 2011, 21, 11556-11565.

32. Chin, P. T. K.; Donegá, C. d. M.; van Bavel, S. S.; Meskers, S. C. J.; Sommerdijk, N. A. J. M.; Janssen, R. A. J. Highly Luminescent $\mathrm{CdTe} / \mathrm{CdSe}$ Colloidal Heteronanocrystals with Temperature-Dependent Emission Color. J. Am. Chem. Soc. 2007, 129, 14880-14886.

33. Norris, D. J.; Efros, A. L.; Rosen, M.; Bawendi, M. G. Size Dependence of Exciton Fine Structure in CdSe Quantum Dots. Phys. Rev. B 1996, 53, 16347-16354.

34. van Dijk, J. M. F.; Schuurmans, M. F. H. On the Nonradiative and Radiative Decay Rates and a Modified Exponential Energy Gap Law for $4 \mathrm{f}-4 \mathrm{f}$ Transitions in Rare-Earth lons. J. Chem. Phys. 1983, 78, 5317-5323.

35. Tilstra, L. G.; Arts, A. F. M.; de Wijn, H. W. Optically Excited Ruby as a Saser: Experiment and Theory. Phys. Rev. B 2007, 76, 024302.

36. Camargo, P. H.; Lee, Y. H.; Jeong, U.; Zou, Z.; Xia, Y. Cation Exchange: A Simple and Versatile Route to Inorganic Colloidal Spheres with the Same Size but Different Compositions and Properties. Langmuir 2007, 23, 2985-2992.

37. Miszta, K.; Dorfs, D.; Genovese, A.; Kim, M. R.; Manna, L. Cation Exchange Reactions in Colloidal Branched Nanocrystals. ACS Nano 2011, 5, 7176-7183.

38. Bhattacharya, S; Estrin, Y.; Moshe, O.; Rich, D. H.; Solovyov, L. A.; Gedanken, A. Highly Luminescent $\mathrm{Zn}_{x} \mathrm{Cd}_{1-x} / \mathrm{C}$ Core/ Shell Nanocrystals: Large Scale Synthesis, Structural and Cathodoluminescence Studies. ACS Nano 2009, 3, 18541876.

39. Kim, J. C.; Rho, H.; Smith, L. M.; Jackson, H. E.; Lee, S.; Dobrowolska, M.; Furdyna, J. K. Temperature Dependent Micro-Photoluminescence of Individual CdSe Self-Assembled Quantum Dots. Appl. Phys. Lett. 1999, 75, 214216.

40. Valerini, D.; Creti, A.; Lomascolo, M.; Manna, L.; Cingolani, R.; Anni, M. Temperature Dependence of the Photoluminescence Properties of Colloidal CdSe/ZnS Core/Shell Quantum Dots Embedded in a Polystyrene Matrix. Phys. Rev. B 2005, 71, 235409.

41. Knappenberger, K. L.; Wong, D. B.; Xu, W.; Schwartzberg, A. M.; Wolcott, A.; Zhang, J. Z.; Leone, S. R. ExcitationWavelength Dependence of Fluorescence Intermittency in CdSe Nanorods. ACS Nano 2008, 2, 2143-2153.

42. Baranov, D.; Fiore, A.; van Huis, M.; Giannini, C.; Falqui, A.; Lafont, U.; Zandbergen, H.; Zanella, M.; Cingolani, R.; Manna, L. Assembly of Colloidal Semiconductor Nanorods in Solution by Depletion Attraction. Nano Lett. 2010, 10, 743-749.

43. Zhang, W.; Chen, G.; Wang, J.; Ye, B. C.; Zhong, X. Design and Synthesis of Highly Luminescent Near-Infrared-Emitting Water-Soluble CdTe/CdSe/ZnS Core/Shell/Shell Quantum Dots. Inorg. Chem. 2009, 48, 9723-9731.

44. Bomm, J.; Buchtemann, A.; Fiore, A.; Manna, L.; Nelson, J. H.; Hill, D.; van Sark, W. G. Fabrication and Spectroscopic Studies on Highly Luminescent CdSe/CdS Nanorod Polymer Composites. Beilstein J. Nanotechnol. 2010, 1, 94-100. 